\title{
Use of Maximum Likelihood-Mixed Models to select stable reference genes: a case of heat stress response in sheep
}

\author{
Magdalena Serrano ${ }^{1 *}$, Natalia Moreno-Sánchez ${ }^{1}$, Carmen González', Ane Marcos-Carcavilla', Mario Van Poucke², \\ Jorge H Calvo ${ }^{3}$, Judit Salces ${ }^{1}$, Jaime Cubero ${ }^{4}$ and María J Carabaño ${ }^{1}$
}

\begin{abstract}
Background: Reference genes with stable expression are required to normalize expression differences of target genes in qPCR experiments. Several procedures and companion software have been proposed to find the most stable genes. Model based procedures are attractive because they provide a solid statistical framework. NormFinder, a widely used software, uses a model based method. The pairwise comparison procedure implemented in GeNorm is a simpler procedure but one of the most extensively used. In the present work a statistical approach based in Maximum Likelihood estimation under mixed models was tested and compared with NormFinder and geNorm softwares. Sixteen candidate genes were tested in whole blood samples from control and heat stressed sheep.

Results: A model including gene and treatment as fixed effects, sample (animal), gene by treatment, gene by sample and treatment by sample interactions as random effects with heteroskedastic residual variance in gene by treatment levels was selected using goodness of fit and predictive ability criteria among a variety of models. Mean Square Error obtained under the selected model was used as indicator of gene expression stability. Genes top and bottom ranked by the three approaches were similar; however, notable differences for the best pair of genes selected for each method and the remaining genes of the rankings were shown. Differences among the expression values of normalized targets for each statistical approach were also found.

Conclusions: Optimal statistical properties of Maximum Likelihood estimation joined to mixed model flexibility allow for more accurate estimation of expression stability of genes under many different situations. Accurate selection of reference genes has a direct impact over the normalized expression values of a given target gene. This may be critical when the aim of the study is to compare expression rate differences among samples under different environmental conditions, tissues, cell types or genotypes. To select reference genes not only statistical but also functional and biological criteria should be considered. Under the method here proposed SDHAMDH1 have arisen as the best set of reference genes to be used in GPCR assays to study heat shock in ovine blood samples.
\end{abstract}

\section{Background}

Quantitative real-time PCR (qPCR) has become a widely used method for both quantitative and qualitative determination of molecular targets. Reliable quantitative expression measurements depend on controlling several parameters (initial sample amount, efficiency of cDNA

\footnotetext{
* Correspondence: malena@inia.es

'Departamento de Mejora Genética Animal, INIA, Ctra. de la Coruña km 7.5, Madrid, Spain

Full list of author information is available at the end of the article
}

synthesis, etc). Usually, these parameters are normalized by means of one or more reference gene(s) (RGs), whose expression is supposed to remain stable in all the tissues and cells or along the different conditions under investigation. However, since the expression of some if not all RGs varies depending on biological samples [1] the use of RGs is controversial. Lee and co-workers [2] proposed that all genes are differentially expressed in at least one biological context, so the expression of every gene would be context dependent. Therefore, following

\section{Biomed Central}


the indications proposed by the MIQE (Minimum Information for Publication of Quantitative Real-Time PCR Experiments) guidelines [3] the utility of chosen reference genes must be confirmed by each research group for every experimental setup [4].

The use of proper mathematical methods to estimate the expression stability of genes under specific conditions is one of the most critical points in the search of RGs. In the last years, some efforts have been made to determine the best way to estimate expression stability of candidate reference genes. Concerning the former, the Pair-Wise comparison method employed by geNorm [4], the most used software in the establishment of RGs, considers that all the samples belong to one group (e.g. tissue, treatment, environmental conditions, etc), and conversely the estimate of the expression stability ignores differences in gene expression level and gene expression variability across groups. On the other hand, Andersen et al. [5] proposed a model-based approach to identify RGs, by means of the NormFinder Visual Basic application for Microsoft Excel. This method estimates the intra- and inter-group variances for each gene and calculates a stability value by combining both sources of variation. Another model-based procedure is the method proposed by Szabo et al. [6] which also uses the intraand inter-gene variation across groups but in a more solid statistical framework. In this case, the stability criterion is the Mean Square Error (MSE), a measure of variability around an intended value, the overall expression level of a gene. Abruzzo et al [7] compared several models including the fixed effect models used in [6] and [5] and models with random effects, showing preference for a model with random gene by group interaction. A model based procedure using mixed models and optimal statistical methods, such as Maximum Likelihood, to estimate inter- and intra-group variances would then be desirable.

Our biological example is the case of the heat-stress response in the ovine species. Variations in the environmental temperature usually stress the organism and may result in the evolution of adaptative genetic mechanisms to cope with extreme temperatures. At cellular level, heat-shock lead to changes in gene expression in most (if not all) cells as well in a variety of organs and tissues associated with the acclimation response. There are some studies about gene expression under heat stress situations in species such as Dinoflagellates [8], Shrimps [9] and Honey bees [10] in which Cal, Rp-S4, SAM and Tub; RPS18; and rp49 have been used as RGs, respectively. De Boer et al. [11] developed a study to detect RGs for various stress conditions in soil arthropods. They found SDHA, YWHAZ and ACTB among the most stable genes under heat stress conditions. However, the suitability of genes for qPCR normalization in the field of adaptation to different thermal conditions in mammals, and particularly in the ovine species, remains unsolved.

In this study, we propose a Maximum Likelihood (ML) Mixed model based approach to estimate the expression stability of 16 candidate RGs taking the heat stress response in the ovine species as example. A comparison with other classical approaches widely used in qPCR experiments was also performed. To test the impact of using alternative methods, normalization factors were calculated with the Delta $\mathrm{Ct}$ method for the reference genes selected with each approach and used to normalize some target genes.

\section{Results}

\section{Gene expression and qPCR efficiencies}

Figure 1 shows the distribution of the Quantification Cycle $(\mathrm{Cq})$ values for the 16 genes tested. The highest expression rate was found for RPS18, with an average Cq value of 13.3. On the contrary, ACACA had the highest average Cq value (34.6), which indicated its low expression rate in leukocytes.

qPCR efficiencies (E) of the candidate RGs are shown in Table 1. Due to technical difficulties, CYP1A1 efficiency could not be estimated and its value (1.99) was taken from the literature [12]. Amplification E ranged from $74 \%$ to $98 \%$. Higher $\mathrm{E}$ were found for candidate genes included in the Sheep geNorm kit (from 1.84 to 1.98), since primers and the PCR conditions must be designed to amplify sequences with $\mathrm{E}$ close to 2 . Genes from Lampo et al. [13] showed lower E (from 1.74 to 1.77) probably because their PCR amplification conditions are different from the standard ones of the kit. Most qPCR studies use the $\log _{2}$ transformed Cycle Threshold or Crossing Point (named Cq according to MIQE guidelines [3]), as the expression rate variable, assuming that the $\mathrm{E}$ is maximum $(100 \%)$ and therefore $E=2$ for all genes. However, Tichopad et al. [14] pointed out that $\mathrm{E}$ evaluation is an essential marker in real-time quantification procedure and therefore corrected models by this parameter are highly recommended.

\section{Maximum Likelihood-Mixed model approach}

Goodness of fit criteria (-2logL (L likelihood), AIC (Akaike's Information Criterion) and BIC (Schwarz's Bayesian Criterion)) and predictive ability (PD) values of the alternative mixed models tested are shown in Table 2. Full models including the three main effects (treatment, gene and sample) and all first order interactions (models 1, 2 and 3 in Table 3 ) showed both, better goodness of fit and lower PD than models that do not considers any of the interactions. This indicates that such interactions were relevant in fitting and predicting 


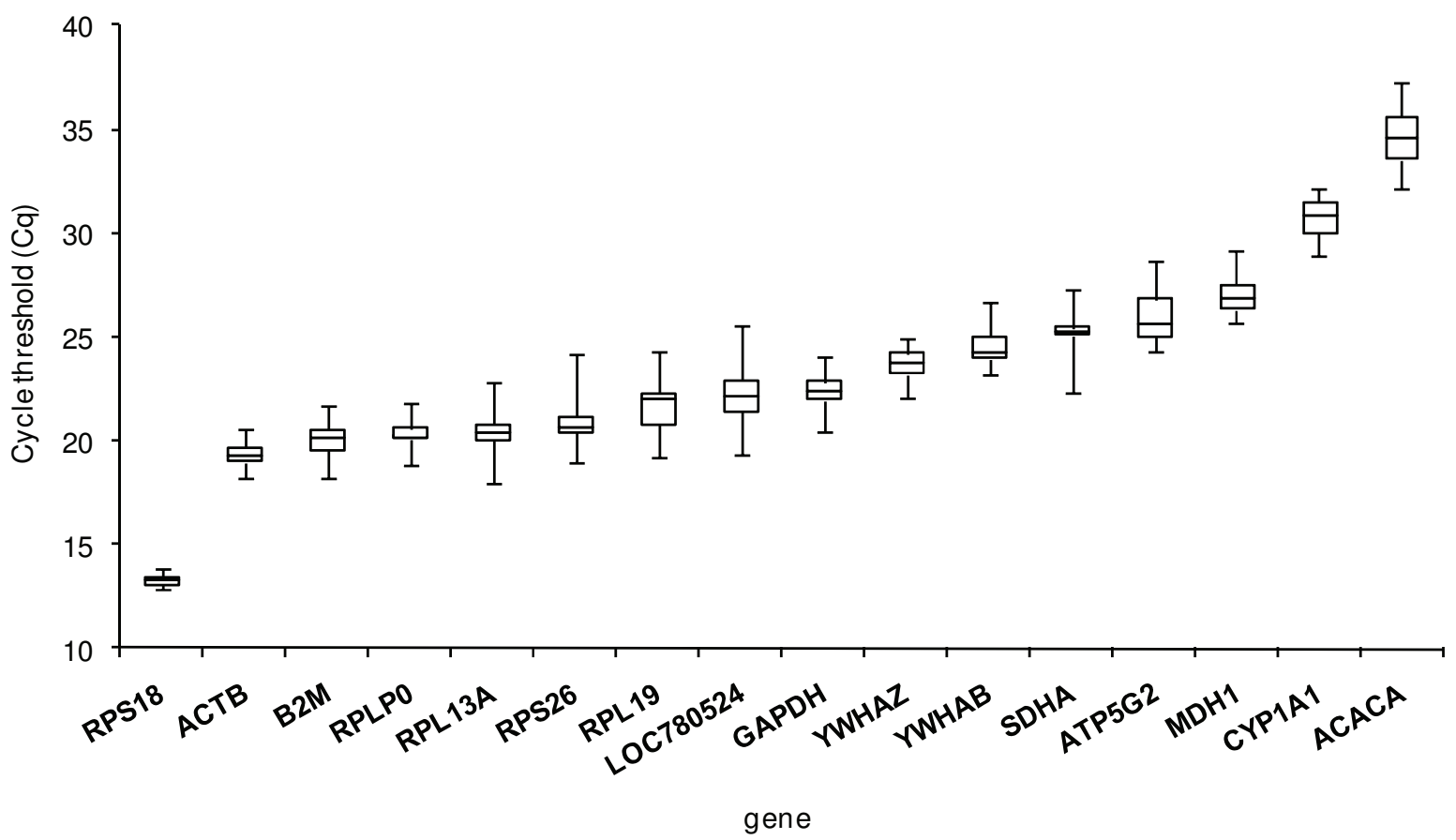

Figure 1 Distribution of Cycle threshold (Cq) values for the candidate reference genes. Distribution of Cycle threshold (Cq) values for the candidate reference genes ( $n=29$ samples) obtained by qPCR. Data of the three replicates per sample were averaged and those of the two thermal treatments were pooled for each gene. Boxes show the range of $\mathrm{Cq}$ values within each gene; the centre line indicates de median; extended vertical bars show standard deviation of the mean.

expression data obtained from different samples and treatments. Model 3 which considered heteroskedastic residual variance linked to the txg effect (32 levels), showed the best goodness of fit (BIC relative to minimum value $=100 \%$; larger is better) and higher $\mathrm{PD}(\mathrm{PD}$

Table 1 Estimated gene amplification efficiencies

\begin{tabular}{lccc}
\hline Gene & slope & Efficiency & correlation \\
\hline RPS18 & -3.593 & 1.90 & 1.000 \\
\hline YWHAZ & -3.377 & 1.98 & 0.995 \\
\hline ACACA & -3.701 & 1.86 & 0.999 \\
\hline RPS26 & -3.701 & 1.86 & 0.999 \\
\hline B2M & -3.661 & 1.88 & 0.999 \\
\hline MDH1 & -3.730 & 1.85 & 0.856 \\
\hline LOC780524 & -3.598 & 1.90 & 0.998 \\
\hline RPL19 & -3.648 & 1.88 & 0.842 \\
\hline ATP5G2 & -3.775 & 1.84 & 0.993 \\
\hline GAPDH & -3.624 & 1.89 & 0.995 \\
\hline CYP1A1 & - & 1.99 & 0.997 \\
\hline ACTB & -3.727 & 1.85 & 0.998 \\
\hline RPL13A & -4.020 & 1.77 & 0.996 \\
\hline RPLPO & -4.161 & 1.74 & 0.997 \\
\hline SDHA & -4.033 & 1.77 & 0.996 \\
\hline YWHAB & -4.138 & 1.74 & 0.996 \\
\hline
\end{tabular}

relative to maximum $=60.1 \%$; smaller is better) than models 1 and 2. Still model 3 showed lower PD than the reduced models (models 4 to 7 ). Model 2, which only differed from model 3 in the residual variance definition (in this case heteroskedastic residual variance was linked to the gene effect, 16 levels), yielded also good fit and predictive parameters $(\mathrm{BIC} \%=95.6 ; \mathrm{PD} \%=52.5)$. On the other hand, model 1 , with homoskedatic residual variance, had the worst goodness of fit $(\mathrm{BIC} \%=87.7)$

Table 2 Goodness of fit and predictive ability criterion of the tested ML-mixed models

\begin{tabular}{|c|c|c|c|c|c|c|c|}
\hline MID & $\begin{array}{c}\mathrm{n}^{\circ} \\
\text { parameters }\end{array}$ & $-2 \log L$ & AIC & $\mathrm{BIC}$ & $\begin{array}{l}\text { BIC } \\
\text { (\%) }\end{array}$ & PD & $\begin{array}{l}\text { PD } \\
(\%)\end{array}$ \\
\hline 1 & 5 & -3141.0 & -3097.0 & -3081.4 & 87.67 & 0.001233 & 52.67 \\
\hline 2 & 20 & -3460.4 & -3386.4 & -3360.2 & 95.60 & 0.001230 & 52.54 \\
\hline 3 & 36 & -3658.2 & -3552.2 & -3514.7 & 100 & 0.001408 & 60.14 \\
\hline 4 & 19 & -3156.9 & -3084.9 & -3059.5 & 87.04 & 0.002027 & 86.58 \\
\hline 5 & 35 & -3203.0 & -3099.0 & -3062.2 & 87.12 & 0.002020 & 86.28 \\
\hline 6 & 19 & -3016.5 & -2944.5 & -2919.1 & 83.05 & 0.001827 & 78.04 \\
\hline 7 & 35 & -3308.0 & -3204.0 & -3167.2 & 90.11 & 0.002341 & 100 \\
\hline 8 & 32 & -3476.8 & -3296.8 & -3164.9 & 90.04 & 0.002084 & 89.02 \\
\hline
\end{tabular}


Table 3 ML-Mixed models description

\begin{tabular}{|c|c|c|c|}
\hline MID & Model & Variance structure & $\mathrm{n}^{\circ}$ (co)variance parameters \\
\hline 1 & $y_{i j k}=\mu+t_{i}+g_{j}+a_{k}+t g_{i j}+t a_{i k}+g a_{j k}+e_{i j k}$ & $\mathrm{a} \sim N\left(0, \sigma_{\mathrm{a}}^{2}\right) ; \operatorname{tg} \sim N\left(0, \sigma_{\mathrm{tg}}^{2}\right) ; \operatorname{ta} \sim N\left(0, \sigma_{\mathrm{ta}}^{2}\right) ; \mathrm{ga} \sim N\left(0, \sigma_{\mathrm{ga}}^{2}\right) ; \mathrm{e} \sim N\left(0, \sigma^{2} \mathrm{e}\right)$ & 5 \\
\hline 2 & $y_{i j k}=\mu+t_{i}+g_{j}+a_{k}+t g_{i j}+t a_{i k}+g a_{j k}+e_{i j k}$ & $\mathrm{a} \sim N\left(0, \sigma_{a}^{2}\right) ; \operatorname{tg} \sim N\left(0, \sigma_{\text {tg }}^{2}\right) ; \operatorname{ta} \sim N\left(0, \sigma_{\text {ta }}^{2}\right) ; \operatorname{ga} \sim N\left(0, \sigma_{g a}^{2}\right) ; e \sim N\left(0, \sigma^{2} e_{j}\right)$ & 20 \\
\hline 3 & $y_{i j k}=\mu+t_{i}+g_{j}+a_{k}+t g_{i j}+t a_{i k}+g a_{j k}+e_{i j k}$ & $\mathrm{a} \sim N\left(0, \sigma_{a}^{2}\right) ; \operatorname{tg} \sim N\left(0, \sigma_{\text {tg }}^{2}\right) ; \operatorname{ta} \sim N\left(0, \sigma_{\text {ta }}^{2}\right) ; g a \sim N\left(0, \sigma_{g a}^{2}\right) ; e \sim N\left(0, \sigma^{2} e_{i j}\right)$ & 36 \\
\hline 4 & $y_{i j k}=\mu+t_{i}+g_{j}+a_{k}+t g_{i j}+t a_{i k}+e_{i j k}$ & $\mathrm{a} \sim N\left(0, \sigma_{\mathrm{a}}^{2}\right) ; \operatorname{tg} \sim N\left(0, \sigma_{\mathrm{tg}}^{2}\right) ; \operatorname{ta} \sim N\left(0, \sigma_{\mathrm{ta}}^{2}\right) ; \mathrm{e} \sim N\left(0, \sigma^{2} \mathrm{e}_{\mathrm{j}}\right)$ & 19 \\
\hline 5 & $y_{i j k}=\mu+t_{i}+g_{j}+a_{k}+t g_{i j}+t a_{i k}+e_{i j k}$ & $\mathrm{a} \sim \mathrm{N}\left(0, \sigma_{\mathrm{a}}^{2}\right) ; \operatorname{tg} \sim \mathrm{N}\left(0, \sigma_{\mathrm{tg}}^{2}\right) ; \operatorname{ta} \sim \mathrm{N}\left(0, \sigma_{\mathrm{ta}}^{2}\right) ; \mathrm{e} \sim \mathrm{N}\left(0, \sigma^{2} \mathrm{e}_{\mathrm{ij}}\right)$ & 35 \\
\hline 6 & $y_{i j k}=\mu+t_{i}+g_{j}+a_{k}+t_{i j}+g a_{i k}+e_{i j k}$ & $\mathrm{a} \sim \mathrm{N}\left(0, \sigma_{\mathrm{a}}^{2}\right) ; \operatorname{tg} \sim N\left(0, \sigma_{\text {tg }}^{2}\right) ; \operatorname{ga} \sim N\left(0, \sigma_{g a}^{2}\right) ; e \sim N\left(0, \sigma^{2} e_{j}\right)$ & 19 \\
\hline 7 & $y_{i j k}=\mu+t_{i}+g_{j}+a_{k}+\operatorname{tg}_{i j}+g a_{i k}+e_{i j k}$ & $\mathrm{a} \sim N\left(0, \sigma_{\mathrm{a}}^{2}\right) ; \operatorname{tg} \sim N\left(0, \sigma_{\mathrm{tg}}^{2}\right) ; \mathrm{ga} \sim N\left(0, \sigma_{\mathrm{ga}}^{2}\right) ; \mathrm{e} \sim \mathrm{N}\left(0, \sigma^{2} \mathrm{e}_{\mathrm{ij}}\right)$ & 35 \\
\hline 8 & $y_{i j k}=\mu+\operatorname{tg}_{i j}+t a_{i k}+e_{i j k}$ & $\mathrm{e} \sim \mathrm{N}\left(0, \sigma^{2} \mathrm{e}_{\mathrm{ij}}\right)$ & 32 \\
\hline
\end{tabular}

and was similar to model 2 in PD (PD\% = 52.7). Model 8 , which mimics the approach proposed by Andersen et al. [5] showed good behavior in fitting data $(\mathrm{BIC} \%=$ 90.04) but very large predicting ability (PD\% = 89.02).

Considering these results, models 2 and 3 were selected to estimate the stability value (MSE) of the 16 candidate RGs. For each gene, two MSE values, one for each treatment, were calculated. Table 4 shows estimates of bias, variances and MSE of the candidate RGs obtained with mixed model 3 . Since results from models 2 and 3 were similar, only those from model 3 are

\begin{tabular}{|c|c|c|c|c|c|c|}
\hline \multirow[t]{2}{*}{ Gene } & \multicolumn{2}{|r|}{ Variance } & \multirow{2}{*}{$\begin{array}{r}\text { Variance } \\
\text { Heat } \\
\text { stress }\end{array}$} & \multirow{2}{*}{$\begin{array}{r}\text { MSE } \\
\text { Control }\end{array}$} & \multirow{2}{*}{$\begin{array}{r}\text { MSE } \\
\begin{array}{r}\text { Heat } \\
\text { stress }\end{array}\end{array}$} & \multirow{2}{*}{$\frac{\text { MSE }}{\max }$} \\
\hline & Bias & Control & & & & \\
\hline RPS18 & 0.00652 & 0.00063 & 0.00002 & 0.00067 & 0.00006 & 0.00067 \\
\hline RPS26 & 0.01708 & 0.00059 & 0.00006 & 0.00088 & 0.00035 & 0.00088 \\
\hline SDHA & -0.01632 & 0.00006 & 0.00087 & 0.00033 & 0.00114 & 0.00114 \\
\hline$B 2 M$ & 0.00753 & 0.00008 & 0.00143 & 0.00014 & 0.00148 & 0.00148 \\
\hline$\overline{A C T B}$ & -0.02230 & 0.00118 & 0.00002 & 0.00168 & 0.00052 & 0.00168 \\
\hline $\mathrm{MDH1}$ & 0.03549 & 0.00065 & 0.00009 & 0.00191 & 0.00135 & 0.00191 \\
\hline YWHAZ & -0.01932 & 0.00158 & 0.00069 & 0.00196 & 0.00106 & 0.00196 \\
\hline LOC780524 & 0.04607 & 0.00060 & 0.00007 & 0.00272 & 0.00219 & 0.00272 \\
\hline GAPDH & -0.02412 & 0.00243 & 0.00002 & 0.00302 & 0.00060 & 0.00302 \\
\hline$\overline{Y W H A B}$ & -0.04385 & 0.00010 & 0.00139 & 0.00203 & 0.00331 & 0.00331 \\
\hline RPLPO & -0.01210 & 0.00009 & 0.00362 & 0.00023 & 0.00377 & 0.00377 \\
\hline RPL19 & 0.05618 & 0.00002 & 0.00073 & 0.00318 & 0.00389 & 0.00389 \\
\hline$\overline{\text { ATP5G2 }}$ & 0.06370 & 0.00163 & 0.00004 & 0.00569 & 0.00410 & 0.00569 \\
\hline CYP1A1 & -0.02738 & 0.00188 & 0.00553 & 0.00263 & 0.00628 & 0.00628 \\
\hline$\overline{A C A C A}$ & -0.02782 & 0.00613 & 0.00409 & 0.00691 & 0.00486 & 0.00691 \\
\hline RPL13A & -0.03935 & 0.00005 & 0.00628 & 0.00160 & 0.00783 & 0.00783 \\
\hline
\end{tabular}

Bias of the control samples. Estimates of bias of the heat-stress samples were the same as for control but with opposite sign. shown. RPS18 and B2M showed the lowest bias, (small deviation of the expression in each treatment from the overall expression of the gene) which is indicative of the stability of these genes under heat stress conditions. On the contrary, LOC780524, RPL19 and ATP5G2, showed the highest bias values. Although some genes (ATP5G2, $G A P D H$ and $A C T B$ ) showed higher variances in the control than in the stressed samples, or in the other way around ( $R P L 13 A$ and $R P L P O)$, the overall variances of gene expression under both control and heat-stress were small, as expected for candidate RGs.

To rank genes on the basis of their stability, the minimax MSE criterion of Szabo et al. [6] was applied. Briefly, the gene with the minimum MSE value was selected as the most stable. The MSE value for each gene was the largest of the two MSE values for each treatment. Overall, RPS18 was the most stable gene and RPL13A the least.

\section{Comparison with geNorm and NormFinder}

Table 5 shows gene rankings in the basis of our mixed model 3, the NormFinder Model-Based method [5] and the geNorm Pair-Wise comparison method [4]. For the geNorm approach, the stability values $M$ calculated by this software for each gene was used to establish the ranking. Spearman rank correlation between classifications from mixed model 3 and from the other two procedures was 0.66 for NormFinder and 0.62 for geNorm. Rank correlation between NormFinder and geNorm classifications was 0.79 . Although the three rankings were different, some coincidences were found in the first five positions. Thus, RPS18, SDHA and $B 2 M$ were shared among all methods, while $A C T B$ and $Y W H A B$ only between two of them. Similarly, ACACA, ATP5G2 and RPL19 were among the least stable genes under the three approaches. On the other hand, the position of some genes (i.e. $Y W H A B$ and RPL13A) was considerably different depending on the approach. 
Table 5 Ranking of genes based on ML-mixed model 3, NormFinder and geNorm approaches.

\begin{tabular}{ccccccc}
\hline Ranking position & gene & ML-Mixed model 3 & gene & NormFinder & gene & geNorm \\
\hline best pair & RPS26/SDHA & 0.0005 & RPS26/YWHAB & 0.008 & ACTB/RPL13A & 0.294 \\
\hline 1 & RPS18 & 0.0007 & RPS18 & 0.013 & YWHAB & 0.782 \\
\hline 2 & RPS26 & 0.0009 & B2M & 0.022 & RPS18 & 0.790 \\
\hline 3 & SDHA & 0.0011 & YWHAZ & 0.024 & ACTB & 0.796 \\
\hline 4 & B2M & 0.0015 & SDHA & 0.026 & SDHA & 0.797 \\
\hline 5 & ACTB & 0.0017 & YWHAB & 0.029 & B2M & 0.815 \\
\hline 6 & MDH1 & 0.0019 & RPS26 & 0.030 & YWHAZ & 0.833 \\
\hline 7 & YWHAZ & 0.0020 & ACTB & 0.035 & RPL13A & 0.839 \\
\hline 8 & LOC780524 & 0.0027 & CYP1A1 & 0.037 & RPS26 & 0.847 \\
\hline 9 & GAPDH & 0.0030 & GAPDH & 0.039 & GAPDH & 0.882 \\
\hline 10 & YWHAB & 0.0033 & RPL13A & 0.043 & RPLP0 & 0.899 \\
\hline 11 & RPLP0 & 0.0038 & MDH1 & 0.048 & LOC780524 & 1.085 \\
\hline 12 & RPL19 & 0.0039 & ACACA & 0.057 & MDH1 & 1.119 \\
\hline 13 & ATP5G2 & 0.0057 & RPLP0 & 0.058 & RPL19 & 1.140 \\
\hline 14 & CYP1A1 & 0.0063 & LOC780524 & 0.061 & CYP1A1 & 1.170 \\
\hline 15 & ACACA & 0.0069 & RPL19 & 0.072 & ATP5G2 & ACACA
\end{tabular}

NormFinder and mixed model 3 are further compared in Figure 2, where inter- and intra-group variabilities are presented for both procedures. With the exception of $Y W H A B$ and RPL13A, inter-group differences (controlheat stress) estimated following both procedures were similar for most of the genes. Those genes which were over-expressed in heat stress (i.e. $A C A C A, C Y P 1 A 1$, $G A P D H)$ conditions, showed negative inter-group differences. Conversely, those genes that were down-regulated in heat stress (i.e. LOC780524, ATP5G2 and RPL19) showed positive inter-group differences. It is important to highlight that the simultaneous use of genes with positive and negative values would yield lower MSE and NormFinder stability values than sets involving genes with the same sign, due to compensating effects.

\section{Optimal set of RGs}

Best pairs of stable genes for all approaches are shown in the first line of Table 5. In the geNorm approach, stability value of the best pair of genes corresponded to that obtained after several steps of stepwise exclusion of the least stable gene until only two genes from the whole set of sixteen remain. These pairs were different in every approach, RPS26/SDHA for mixed model 3, RPS26/YWHAB for NormFinder and ACTB/RPL13A for geNorm. Under mixed model 3, average MSE for all possible combinations of genes from 2 to 16 (65.519 combinations) were explored to determine the best set of stable genes to be used as normalizers. Table 6 shows the average MSE of the best combinations of sets from 2 to 7 genes and also for the set including all genes.
The RPS18/RPS26/SDHA set had the lowest average MSE (0.00043), revealing the best combination of normalizers. Similarly, RPS26/SDHA and RPS18/SDHA pairs had average MSE values of 0.00047 . Additional file 1 shows the optimal number of control genes for normalization estimated by the geNorm. geNorm authors [4] recommend a cut-off value of 0.15 , thus, the group of two genes (0.100) was the best combination (see 2 vs. 3 genes).

\section{Discussion}

\section{ML-Mixed model approach}

The stability ranking generated by mixed model 3 (Table 5) placed RPS18, RPS26, SDHA, B2M and ACTB in the top five positions. However, as previously mentioned, the selection of RGs has to be based not only on the estimated stability of gene expression, but also on biological and functional criteria. For instance, despite the fact that RPS18 showed a high stability, some works indicate that it is not a good control gene $[15,16]$ because its transcription is carried out by RNA polymerase I, and for accurate quantification by qPCR the use of RGs following the same type of transcription pathways is important. RPS26 encodes the $40 \mathrm{~S}$ ribosomal protein S26. S26 mRNA half-life is more than 20 hours and was found to be expressed at high and comparable levels in various adult human tissues [17]. However, RPS26 has multiple processed pseudogenes dispersed through the genome $[18,19]$. Primers are usually designed to overlap two or more exons in order to distinguish PCR products derived from genomic DNA and RNA. If some of these 


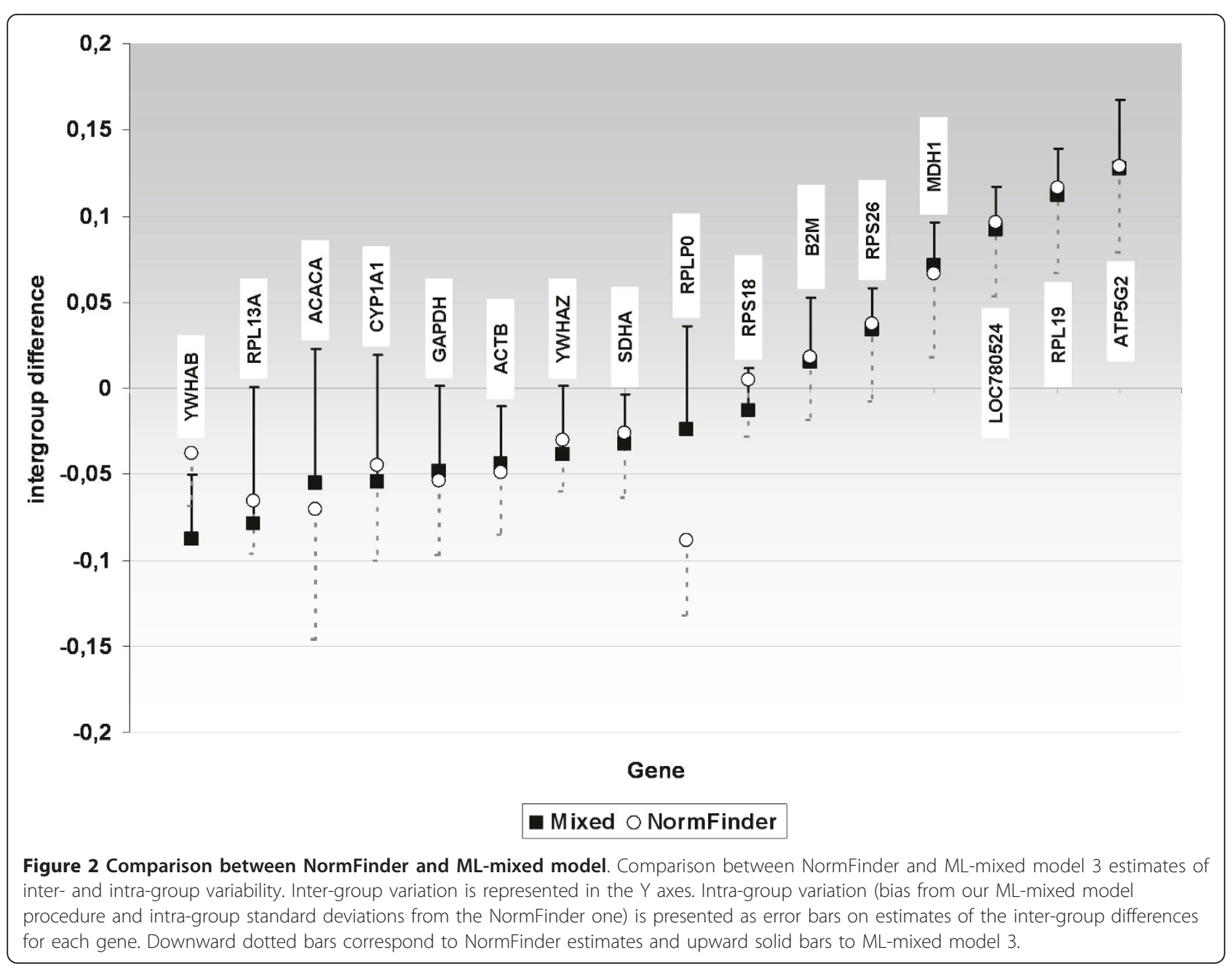

pseudogenes are expressed either constitutively or under particular circumstances, several sequences from different genes could be co-amplified leading to wrong results.

SDHA encodes the succinate dehydrogenase complex subunit A flavoprotein which converts succinate to fumarate as part of the Citric Acid Cycle. Under mixed model 3 this gene showed similar and small variances in control (0.00006) and heat stressed (0.00087) samples, appearing in the $3^{\text {rd }}$ position of the stability ranking. This result is in agreement with other studies which have pointed out SDHA as a good RG to be used in different circumstances, including heat stress in several species, [20]. Beta-2 microglobulin is a component of MHC class I molecules gene and is encoded by $B 2 M$, which has been extensively used as endogenous control in many publications. However, its expression varies considerably under different experimental conditions and therefore its use for normalization is limited. Under mixed model 3, $B 2 M$ showed higher variance in heat stress conditions (0.00142) than under mild temperatures (0.00008).
However since its bias was among the lowest (0.0075), it can be considered as a good RG to study heat stress in ovine blood samples.

$A C T B$ is another commonly used RG in qPCR assays. Its expression has been shown to vary in a tissue-specific and time-dependent manner [12]. However, our results are in line with other studies reporting stable expression of ACTB under temperature stress in Orchesella cincta [11]. Although under our ML-mixed model approach $A C T B$ is placed among the top five stable genes, this was not the case when comparing sets of two or three genes.

Conversely, $M D H 1$ took the $6^{\text {th }}$ place in the mixed model 3 stability ranking, but appeared among the genes constituting the most stable sets. $M D H 1$ encodes the cytosolic malate dehydrogenase which catalyzes the reversible oxidation of malate to oxaloacetate, utilizing the NAD/NADH cofactor system in the citric acid cycle. Cellular localization studies indicate that $M D H 1$ mRNA expression has a strong tissue-specific distribution, being expressed primarily in cardiac and skeletal muscle 
Table 6 Average MSE of the best genes combination for sets from 2 to 7 genes tested under ML-mixed model 3

\begin{tabular}{|c|c|c|c|c|c|c|c|}
\hline $\begin{array}{l}\text { Gene } \\
\text { set }\end{array}$ & & & & & & & $\begin{array}{c}\text { Average } \\
\text { MSE }\end{array}$ \\
\hline 2 & RPS26 & SDHA & & & & & 0.00047 \\
\hline \multirow[t]{7}{*}{$120^{*}$} & RPS18 & SDHA & & & & & 0.00047 \\
\hline & $\mathrm{MDH} 1$ & SDHA & & & & & 0.00057 \\
\hline & $\mathrm{B} 2 \mathrm{M}$ & ACTB & & & & & 0.00077 \\
\hline & $\mathrm{MDH} 1$ & ACTB & & & & & 0.00096 \\
\hline & SDHA & ACTB & & & & & 0.00099 \\
\hline & B2M & SDHA & & & & & 0.00116 \\
\hline & B2M & $\mathrm{MDH} 1$ & & & & & 0.00122 \\
\hline 3 & RPS18 & RPS26 & SDHA & & & & 0.00043 \\
\hline \multirow[t]{7}{*}{560} & RPS18 & LOC780524 & YWHAB & & & & 0.00050 \\
\hline & RPS18 & $\mathrm{MDH1}$ & YWHAB & & & & 0.00050 \\
\hline & RPL19 & ACTB & SDHA & & & & 0.00057 \\
\hline & $\mathrm{MDH} 1$ & SDHA & ACTB & & & & 0.00063 \\
\hline & $\mathrm{MDH} 1$ & B2M & ACTB & & & & 0.00068 \\
\hline & $\mathrm{MDH} 1$ & SDHA & $\mathrm{B} 2 \mathrm{M}$ & & & & 0.00087 \\
\hline & SDHA & ACTB & B2M & & & & 0.00088 \\
\hline 4 & RPS18 & RPS26 & $\mathrm{MDH} 1$ & YWHAB & & & 0.00051 \\
\hline \multirow[t]{2}{*}{1,820} & RPS18 & RPL19 & ACTB & SDHA & & & 0.00051 \\
\hline & RPS18 & RPS26 & LOC780524 & YWHAB & & & 0.00052 \\
\hline 5 & RPS18 & RPS26 & LOC780524 & SDHA & YWHAB & & 0.00048 \\
\hline \multirow[t]{2}{*}{4,368} & RPS18 & RPS26 & $\mathrm{MDH1}$ & SDHA & YWHAB & & 0.00049 \\
\hline & RPS18 & RPS26 & B2M & ACTB & SDHA & & 0.00051 \\
\hline 6 & RPS18 & RPS26 & $\mathrm{MDH} 1$ & LOC780524 & SDHA & YWHAB & 0.00049 \\
\hline \multirow[t]{2}{*}{8,008} & RPS18 & RPS26 & RPL19 & ACTB & SDHA & YWHAB & 0.00052 \\
\hline & RPS18 & $\mathrm{MDH} 1$ & RPL19 & ACTB & SDHA & YWHAB & 0.00053 \\
\hline 7 & RPS18 & RPS26 & $\mathrm{MDH} 1$ & RPL19 & ACTB & SDHA & 0.00048 \\
\hline \multirow[t]{2}{*}{11,440} & RPS18 & RPS26 & LOC780524 & RPL19 & ACTB & SDHA & 0.00049 \\
\hline & RPS18 & $\mathrm{MDH} 1$ & LOC780524 & RPL19 & ACTB & SDHA & 0.00054 \\
\hline All genes & & & & & & & 0.00156 \\
\hline
\end{tabular}

*Under each set number (from 2 to 7 ) the number of possible combinations appears.

and in the brain, at intermediate levels in the spleen, kidney, intestine, liver and testes and at low levels in lung and bone marrow [21]. This study demonstrated that $M D H 1$ expression was stable under different temperature conditions in ovine whole blood samples, and points toward this gene as a suitable RG to be used in expression studies addressing the heat stress response in sheep.

\section{Comparison of methods}

The top five positions of the stability rankings obtained with the mixed model 3 , NormFinder and geNorm approaches (Table 5) contained the RPS18, SDHA and $B 2 M$ candidates. This shows the accordance of the three methods to identify highly stable genes even when different calculation methods are used. However, for the remaining genes there were many larger differences among the three rankings.

Model based procedures such as the ones proposed by Andersen et al. [5-7] and Abruzzo et al. [6] are appealing. They provide a solid statistical framework which allows accounting for different sources of variation (e.g. differences in expression levels across genes, amount of mRNA in the samples, treatments, tissues and cells types, developmental stages, sampling, etc.) in the estimation of the genes stability. The goal is to achieve a prediction of gene stability invariant to a variety of effects, making the set of selected RGs more generally applicable. In our case, a model including sample and its interactions with treatment and gene was deemed more plausible for the data analyzed (using likelihood based criteria) and for data not used in the analysis 
(using cross validation statistics). Accounting for those factors when estimating the parameters of interest (gene by treatment effect and gene-treatment variances in our case) removes noise and provides estimates free of the effects of the specific animals sampled for this experiment. The model underlying the NormFinder application [5] does not consider the interaction between sample and treatment and showed a worse predictive ability, illustrating the interest of adjusting for noise to obtain estimates of gene stability applicable to future samples.

Within the model based methods, several alternatives have been proposed to obtain the stability measure. In NormFinder [5], inter-group variation is computed as the least square estimate of the group by gene interaction and intra-group variability is obtained following the method of moments approach. Those two components are closely linked to the bias and variance components of the MSE calculation of Szabo et al. [6], followed in our study. Figure 2 shows inter-group variation (from NormFinder) and bias (from our ML-mixed model procedure) for all genes, together with the intra-group standard deviations from NormFinder and from our procedure represented by error bars. Except for RPLPO and $Y W H A B$, the inter-group variability estimate obtained by the two procedures was similar for all genes (Pearson correlation coefficient $=0.95)$. However, the intra-group variability was more dissimilar (Pearson correlation coefficient $=0.38$ ). This may be explained by the different estimation methods followed in both procedures (maximum likelihood in our approach and method of moments in NormFinder [5]). Maximum likelihood estimates have well known asymptotically optimal properties in terms of bias and variance while unbiasedness is the only optimal property of methods of moments. Andersen et al. [5] argued that using REML to obtain estimates of intra-group variances yielded similar results in their data set to the method of moments proposed, but, this result cannot be generalized. Another difference between the stability value obtained by NormFinder and the MSE stability value [6] used in our study is how the inter- and intra-group variation are combined. In NormFinder, those two components are combined by adding estimates of the mean and standard deviation of the posterior distribution (following Bayesian terminology) of the deviation of the observed group mean for each gene from the overall expected value for the log-transformed measure of the gene expression. The mean and variance of the distribution of that deviation depends on inter- and intra-group variation, respectively. This results in a stability value where inter- and intra-group variability do not have the same weight. This is in contrast with the MSE situation, where these two components are equally weighted.
Nevertheless, when intra-group variability is close to zero, the weights on both components are equal. In our data set, the estimated intra-group variances for all genes are small (as expected for RGs). We obtained a stability measure by adding the inter-group variation and the average of the square root of the intra-group variations provided in the extended output of NormFinder which was similar to the overall stability value provided by that software (Pearson and Spearman correlations $=0.96$ ).

The use of a more solid statistical framework such as our ML-mixed approach allows for statistical testing of the differences in gene expression across different conditions. This is an alternative way and a double check through statistical validation procedures for the selection of stable genes, as recently recommended Setiawan and Lokman [22]. In our case, a t-test on the estimated gene by treatment effects provided by the SAS mixed models procedure (PROC MIXED) indicated that differences in expression between the two treatments were only significant ( $\mathrm{p}<5 \%$ ) for RPL19 and ATP5G2 which were positioned by the three procedures among the five most instable genes and considered together with $A C A C A$, among the worst. This shows consistency between stability values and statistical validation approaches.

The difference between our procedure and the one followed by Szabo et al. [6] is that the gene by group interaction is treated as a random effect instead of as a fixed factor. Random effects represent random deviations from the expected value of each data due to that effect. In our case, the random treatment by gene interaction can therefore be used as a direct measure of the so called bias in [6].

Compared to model based procedures, the pair-wise comparison approach used in geNorm [4] is easier to apply and does not require normality assumptions (while maximum likelihood model based methods do). Furthermore, it has shown more robust behavior than NormFinder with small sample sizes [23]. Nevertheless, geNorm has the tendency to top rank genes with correlated expression profiles rather than with minimal variation [5] and does not accommodate the existence of different groups of measures in the calculation of the stability value. When different groups of samples exist, independent analyses for each set of data have to be carried out, often yielding different optimal sets of RGs in each group. We have run geNorm for each set of data (control and heat stress) separately (data not shown). Ranking of RGs differed between treatments, but, two genes, $S D H A$ and $B 2 M$ were shared among the top five of both groups. A better agreement was found for the least stable genes (ACACA, ATP5G2, RPL19 and $C Y P 1 A 1)$, which were located in both treatments in the last five positions. Alternatively, the procedures 
proposed by Andersen et al. [5] and our ML-mixed model approach can also provide within group stability values. Ranking differences were also observed for these procedures between treatments. Here the differences were also bigger among the most stable genes than among those genes estimated as the less stable ones. Results from the different methods were more similar in the control (thermo neutral) than in the heat stress environment. Notice that within groups, differences in stability among genes are only associated to changes in intra-group variances. Genes such as $B 2 M$ and $S D H A$, which were classified by our ML-mixed model approach within the top five stable genes, both globally and in the control group, showed larger variability under heat stress, and were classified as the less stable ones under heat conditions. Since in many situations the goal is to use a given set of RGs in the normalization of the expression of target genes across different conditions, an essential requirement for the RGs is stability of their transcriptional levels across those conditions. The within group stability will only be relevant when target genes are compared under the same experimental conditions.

Finally, one constraint of the geNorm and NormFinder softwares come from the fact that they are implemented for the Microsoft Excel application which involves some restriction regarding the number of samples, treatments and genes that can be analyzed simultaneously. Furthermore, the geNorm application, does not allow empty cells, therefore, those samples in which the amplification of one or more genes fails must be eliminated from the analysis.

\section{Best set of RGs}

There is no consensus about the amount of RGs that should be used in expression studies [4,24,25], although in all cases the common objective is to find the best alternative regarding accuracy and technical constraints. We have analyzed in this work all possible sets of genes considering, both stability and biological criteria. As explained above, RPS18 and RPS26 have been discarded from the analysis for functional reasons. With the MLmixed model 3 the best set of two genes was SDHA/ $M D H 1(\mathrm{MSE}=0.0006)$ and the best one of three genes was RPL19/ACTB/SDHA (MSE =0.0006), after discarding these genes. These values were very close to those of the best pair RPS26/SDHA (MSE =0.0005) obtained under our ML-mixed model 3 approach when all candidates were included. However, pairs constituted by $S D H A / B 2 M$ and SDHA/ACTB had larger MSE values (0.0012 and 0.0010 , respectively). For the geNorm approach the best pair of genes was the same, $A C T B /$ $R P L 13 A$, as the one obtained when including all candidates. However, NormFinder selected a different pair of genes B2M/SDHA. One again, ML-mixed model 3 and
NormFinder approaches identify the same gene (SDHA) for the best set of two genes.

\section{Normalization factors and normalized targets}

Normalization factors were calculated by means of the Delta Ct method [4] for the best pair of reference genes selected with each statistical approach, $A C T B / R P L 13 A$, $B 2 M / S D H A$ and $S D H A / M D H 1$ for geNorm, NormFinder and ML-mixed model, respectively (Additional file 2). Two of the less stable genes $C Y P 1 A 1$ and $A C A C A$ were used as targets to study the impact of using different nornalizers. Pearson and Spearman correlations estimates among normalized values of targets ranged from 0.80 to 0.92 for geNorm-NormFinder, from 0.67 to 0.89 for geNorm-ML- mixed model and from 0.92 to 0.98 for NormFinder-Ml mixed model. Model based methods, NormFinder and ML-mixed model showed the highest correlations indicating statistical similarities underlying the estimation of genes stability under both methods. Although correlations among results obtained under all approaches were high, small differences among the normalized expression rates of targets might be critical when the aim is to detect differential expression among samples from different treatments, tissues, cell types or genotypes.

\section{Conclusions}

A ML-mixed model approach has been presented here as a suitable method to select stable genes to be used as RGs in gene expression studies. Optimal statistical properties of ML estimation together with the flexibility of the mixed model allows estimation of gene expression stability under many different situations without constraints in the amount of data, number of genes and number of treatments or tissues tested. A model selection step can also be performed to choose the optimal model to estimate stability values. The use of goodness of fit and predictive ability criteria is recommended because they measure different quality criteria and can provide unequal model rankings.

Although in the present study we have tested several mixed models according to our experimental needs, many other possibilities can be considered to take into account for new biological situations. For instance, in situations such as drug competition in which interaction between treatments must be fitted, or experiments of sequential gene expression along a period of time where the existence of correlation between successive samples needs to be considered by fitting some structure among residuals.

Comparison with two other procedures currently used showed differences in genes ranking according to their stability values, which were mainly explained by the difference in estimates for the within treatment variability. 
Also differences in normalized expression values of targets were found among the three methods tested. Nevertheless, some genes were selected by the three approaches. RPS18, SDHA and B2M were ranked in the first five positions by the three methods. On the last positions, ACACA, ATP5G2 and RPL19 were the least stable genes under the three approaches.

In this work, the pair of genes $S D H A / M D H 1$ is recommended to normalize target genes expression in peripheral whole blood in studies of the heat-stress response in sheep.

\section{Methods}

\section{Selection of RGs}

Sixteen candidate RGs were tested. Twelve of them (RPS18 (Ribosomal protein S18), YWHAZ (Tyrosine 3monooxygenase/tryptophan 5-monooxygenase activation protein, zeta polypeptide), ACACA (Acetyl-CoA carboxylase), RPS26 (Ribosomal protein S26), B2M (Beta-2 microglobulin), MDH1 (Malate dehydrogenase), LOC780524 (Ribosomal protein S2, RPS2), RPL19 (Ribosomal protein L19), ATP5G2 (ATP synthase, H+ transporting, mitochondrial Fo complex, subunit $\mathrm{C} 2$, subunit 9), GAPDH (Glyceraldehyde 3-phosphate dehydrogenase), CYP1A1 (Cytochrome P4501A1) and ACTB (Actin beta)) were included in the Sheep geNorm kit (Primerdesign Ltd, UK). The remaining four genes (RPL13A (Ribosomal protein L13a), RPLPO (Ribosomal protein large P0), SDHA (Succinate dehydrogenase complex, subunit A, flavoprotein) and $Y W H A B$ (Tyrosine 3monooxygenase/tryptophan 5-monooxygenase activation protein, beta polypeptide)) were choosen because they had been tested as RGs in a gene expression study of the ovine PRNP and SPRN [13] genes. Genes, GeneBank accession numbers and gene ontology are listed in Table 7.

\section{Animal samples, total RNA isolation and CDNA synthesis}

Peripheral whole blood samples from 15 rams of the Manchega Spanish sheep breed were collected under two different climatic conditions in a dry region of central Spain (Ciudad Real). The control samples were collected from 15 animals with an environmental temperature of $28.6^{\circ} \mathrm{C}$ and a relative humidity of $52 \%$. The second set of samples, here considered as the heat stress conditions, was collected from the same animals at an environmental temperature and a relative humidity of $34.4^{\circ} \mathrm{C}$ and $35 \%$, respectively (data from Ciudad Real Meteorological Station, coordinates 629 m-38 59N-03 $55 \mathrm{~W})$.

Total RNA was isolated from $10 \mathrm{ml}$ of whole blood using the LeukoLock kit (Ambion, Inc., TX, USA) following manufacturers instructions. The absence of DNA contamination was verified by minus RT control PCR. The quality of the RNA was assessed based on the demonstration of distinct intact $28 \mathrm{~S}$ and $18 \mathrm{~S}$ ribosomal RNA bands. RNA concentration was determined using a NanoDrop ND-1000 UV/Vis spectrophotometer (Nanodrop Technologies, Inc., DE, USA).

cDNA was synthesized using the ImProm-IITM Reverse Transcription System (Promega Corp., WI, USA). In a first step the primer/RNA mix [RNA (500 ng), oligo (dt) 15 primer $(0.5 \mu \mathrm{g} /$ reaction $)$ and random primers $(0.5 \mu \mathrm{g} /$ reaction) in a final volume of $8 \mu \mathrm{l}$ ] was heated $5 \mathrm{~min}$ at $70^{\circ} \mathrm{C}$ and quickly chilled on ice for $5 \mathrm{~min}$. In a second step, the reverse transcription mix [ImProm-II ${ }^{\mathrm{TM}} 5 \times$ reaction buffer $(1.6 \times), \mathrm{MgCl}_{2}(2.8 \mathrm{mM})$, dNTP mix (0.6 $\mathrm{mM}$ each dNTP), 10U of Recombinant RNAsin Ribonuclease Inhibitor and 20U of ImProm-II'TM Reverse Transcription in a final volume of $12 \mu \mathrm{l}$ ] was mixed with the primer/RNA mix in a final volume of $20 \mu \mathrm{l}$ and subjected to the following thermal profile: $5 \mathrm{~min}$ annealing at $25^{\circ} \mathrm{C}, 60 \mathrm{~min}$ first-strand synthesis reaction at $42^{\circ} \mathrm{C}$ and $15 \mathrm{~min}$ inactivation of reverse transcriptase at $70^{\circ} \mathrm{C}$. The final product was stored at $-20^{\circ} \mathrm{C}$.

\section{qPCR}

qPCR amplification reactions were performed in a final volume of $20 \mu \mathrm{l}$ containing $50 \mathrm{ng}$ of cDNA, $10 \mu \mathrm{l}$ of Precision TM 2X qPCR Mastermix with SYBR GREEN (Biomolecular Technologies, Inc., USA) and $300 \mathrm{nM}$ of each primer. Reactions were run in triplicate on an ABI PRISM 7500 Fast Sequence Detector (Applied Biosystems, CA, USA) following the manufacturer's cycling parameters. Dissociation curves were performed for each gene to check primer specificity and to confirm the presence of a unique PCR product. The corresponding mRNA levels were measured and analyzed by the 7500 System Software (Applied Biosystems, CA, USA). The $\mathrm{Cq}$ (threshold cycle) is defined as the number of cycles needed for the fluorescence to reach a specific threshold level of detection and is inversely correlated with the amount of RNA template present in the reaction.

To estimate PCR efficiencies, standard curves based on 5 serial dilutions $(0.08,0.4,2,10$ and $50 \mathrm{ng} / \mu \mathrm{l})$ of a cDNA stock (a cDNA mixture of all samples collected) were also performed. Efficiencies (E) were calculated from the slope of curves using the formula $E=10^{(-1 /}$ slope) $[26,27]$. For all candidate genes plates consisted of 87 wells, derived from 15 RNA samples (one RNA isolation was lost) for each thermal treatment, each with three technical replicates.

\section{Statistical data analysis. Maximum Likelihood Mixed Model approach}

The use of efficiency corrected mathematical models are strongly recommended and leads to more reliable estimates of the 'real expression ratio' compared to non efficiency corrected ones [28]. In order to take the 
Table 7 Tested candidate reference genes

\begin{tabular}{llll}
\hline $\begin{array}{l}\text { Gene } \\
\text { symbol }\end{array}$ & Gene full name & Gene ID & GO \\
\hline RPS18 & Ribosomal protein S18 & 100036761 & Translation \\
\hline YWHAZ & $\begin{array}{l}\text { Tyrosine 3-monooxygenase/tryptophan 5-monooxygenase activation protein, zeta } \\
\text { polypeptide }\end{array}$ & 780452 & mRNA metabolic process \\
\hline ACACA & Acetyl-CoA carboxilase & 443186 & Lipid process \\
\hline RPS26 & Ribosomal protein S26 & 443468 & Translation \\
\hline B2M & Beta-2 microglobulin & 443295 & Immune response \\
\hline MDH1 & Malate dehydrogenase & 443091 & Glycolysis \\
\hline LOC780524 & Ribosomal protein S2 (RPS2) & 780524 & Translation \\
\hline RPL19 & Ribosomal protein L19 & 100270789 & Translation \\
\hline ATP5G2 & ATP synthase, H+ transporting, mitochondrial Fo complex, subunit C2 (subunit 9) & 443542 & Ion transport \\
\hline GAPDH & Glyceraldehyde 3-phosphate dehydrogenase & 443005 & Glycolysis \\
\hline CYP1A1 & Cytochrome P4501A1 & 100170113 Oxidation-reduction process \\
\hline ACTB & Actin beta & 443052 & Cellular component \\
\hline RPL13A & Ribosomal protein L13a & 100036760 & Translation \\
\hline RPLPO & Ribosomal protein large P0 & 100036764 & Translation \\
\hline SDHA & Succinate dehydrogenase complex, subunit A, flavoprotein & 100036762 & Oxidation-reduction process \\
\hline YWHAB & Tyrosine 3-monooxygenase/tryptophan 5-monooxygenase activation protein, beta & 100036763 Protein targeting \\
\hline
\end{tabular}

differences in the amplification efficiency (E) of the qPCR reaction of each gene into account, the $\log _{\mathrm{E}}$ of the raw $\mathrm{Cq}$ data was used as the dependent variable. In this regard, it is important to consider that a commonly used approach is to asume $100 \%$ efficiency $(E=2)$ for all genes. Replicates were included in the model as repeated measures of the same animal which allows for a better correction of technical differences in well's loading.

Different Mixed models were fitted using the MIXED procedure of the SAS/STAT ${ }^{\circledR}$ statistical package. Models included treatment $(\mathrm{t})$ and gene $(\mathrm{g})$ as systematic fixed effects; sample (a), treatment $\times$ gene $(\mathrm{tg})$, treatment $\times$ sample (ta) and gene $\times$ sample (ga) as alternative random variables, and homoskedastic (equal residual variance for all observations) and heteroskedastic (different residual variances for groups of observations pertaining to each class of either the gene or the treatment $\times$ gene effects) residuals. The model proposed by Andersen and coworkers [5] which includes tg and ta as fixed effects and a heteroskedastic residual variance for the tg effect was also tested (model 8). Table 3 shows the equations of the mixed models tested, their variance structure and the number of (co)variance parameters.

The estimation method was Maximum Likelihood (ML). Several statistics were used as Goodness of Fit indicators: -2 Log Likelihood (-2logL, smaller is better), Akaike's Information Criterion (AIC $=-2 \mathrm{l}+2 \mathrm{~d}$, smaller is better), and Schwarz's Bayesian Criterion $(\mathrm{BIC}=-2 \mathrm{l}$ +dlogn, smaller is better), where $l$ is the maximum value of the log likelihood, $\mathrm{d}$ is the dimension of the model and $\mathrm{n}$ is the number of effective observations. Model Predictive Ability was evaluated by crossvalidation using a training set (for estimation of parameters) composed of 917 records ( $2 / 3$ of the whole data available) and a testing set (for prediction of new data) with 460 records (1/3 of the original set of observations). Average square differences (PD) between observed and predicted values were used as models predictive ability criterion. PD\% reflects the percentage of predictive ability loss (smaller is better).

The measure of the gene expression stability was obtained by calculating the Mean Square Error (MSE), as in [6] under the best model selected in the previous step. MSE was defined as:

$$
\text { MSE }=(\text { bias })^{2}+\text { variance }
$$

In this equation, bias was the estimate of the random treatment $\times$ gene interaction and variance was the ML estimate of the variance of the residual term corresponding to the selected model.

To rank genes on the basis of their stability, the minimax MSE criterion of Szabo et al. [6] was performed. In brief, the gene with the smallest value among the largest MSE of the two treatments was selected as the most stable one. To find a set of genes for normalization across treatments, all possible combinations of genes from 2 to 16 were explored. For every combination, 
MSE was calculated by averaging bias and variances of the genes contained in each set.

\section{Other approaches currently used}

In order to contrast the results obtained under our statistical approach to test candidate RGs stability, we run the geNorm [4] and the Normfinder [5] softwares. As both programs rely on the input of relative values, the $\mathrm{Cq}$ of the 3 replicates per sample were averaged in a single value.

For the geNorm approach, sample Cq values were transformed to relative quantities (Q) with the equation:

$$
\mathrm{Q}=\mathrm{E}^{\text {(minCq-sampleCq) }}
$$

where $\mathrm{E}=$ amplification efficiency; $\operatorname{minCq}=$ lowest $\mathrm{Cq}$ value $(\mathrm{Cq}$ value of the sample with the highest expression) and sampleCq $=\mathrm{Cq}$ value of each sample. geNorm is based on the principle that the expression ratio of two ideal RGs is identical in all samples, and defines the $M$ value as the average pair-wise comparison of a gene with all the other tested. Genes with low $M$ values have less variation and more stable expression. Then, the genes are ranked according to their expression stability $M$ value. geNorm does not accommodate the existence of groups (treatments in our case) and therefore samples obtained under heat stress or thermoneutral conditions were treated alike.

For NormFinder, the dependent variable was the $\log _{E}$ of the Cq values as in our ML-mixed model approach. Genes are ranked according to a stability value that combines intra- and inter-group variability obtained from a fixed effects model.

Both softwares were used to determine a ranking of gene expression stability, in order to compare them with the results obtained under our mixed model approach.

Normalization factors were calculated using the Delta Ct method [4] for the best pair of reference genes selected by each approach tested. Expression values of target genes were normalized to test differences from the reference genes used.

\section{Additional material}

Additional file 1: GeNorm optimum number of reference genes. Evaluation of the optimum number of reference genes according to the geNorm software. The magnitude of the change in the normalization factor after the inclusion of an additional gene reflects the improvement obtained.

Additional file 2: Normalization factors. Normalization factors calculated with the Delta Ct method for each of the best pair of reference genes selected by geNorm, NormFinder and ML-mixed model 3.

\section{Acknowledgements}

We thank AGRAMA breeders association, CERSYRA Valdepeñas and INIAMadrid to provide for biological samples. This work was supported by RTA2009-00098-00-00 INIA project.

\section{Author details}

'Departamento de Mejora Genética Animal, INIA, Ctra. de la Coruña km 7.5, Madrid, Spain. '2Department of Nutrition, Genetics and Ethology, Faculty of Veterinary Medicine, Ghent University, Heidestraat 19, B-9820 Merelbeke, Belgium. ${ }^{3}$ Unidad de Tecnología en Producción Animal, CITA, Zaragoza, Spain. ${ }^{4}$ Departamento de Protección Vegetal, INIA. Ctra. de la Coruña km 7.5, Madrid, Spain.

\section{Authors' contributions}

MS conceived of the study, performed the statistical analyses and draft the manuscript. NMS participated in GPCR data analyses and in drafting the manuscript. CG carried out the qPCR assays. AMC has participated in the QPCR assays design, protocols development and in drafting the manuscript. MVP has participated in the GPCR assays design, protocols development and software management. JHC contributed in the experimental design and in results interpretation. JS has processed biological samples and contributed to GPCR assays. JC has contributed to GPCR assays, standard curves design and discussion. MJ participated in statistical analyses design, results interpretation and in drafting the manuscript. All authors read and approved the final manuscript.

Received: 30 March 2011 Accepted: 17 August 2011

Published: 17 August 2011

\section{References}

1. Dheda K, Huggett JF, Bustin SA, Johnson MA, Rook G, Zumlan A: Validation of housekeeping genes for normalizing RNA expression in real-time PCR. BioTechniques 2004, 37:112-119.

2. Lee PD, Sladek R, Greenwood CMT, Hudson TJ: Control Genes and Variability: Absence of Ubiquitous Reference Transcripts in Diverse Mammalian Expression Studies. Genome Res 2002, 12:292-297.

3. Bustin SA, Benes V, Garson JA, Hellemans J, Huggett J, Kubista M, Mueller R, Nolan T, Pfaffl MW, Shipley GL, et al: The MIQE Guidelines: Minimum Information for Publication of Quantitative Real-Time PCR Experiments. Clin Chem 2009, 55(4):611-622.

4. Vandesompele J, De Preter K, Pattyn F, Poppe B, Van Roy N, De Paepe A, Speleman F: Accurate normalization of real-time quantitative RT-PCR data by geometric averaging of multiple internal control genes. Genome Biol 2002, 3(7):research0034-research0034.0011.

5. Andersen $\mathrm{CL}$, Ledet-Jensen J, Ørntoft T: Normalization of real-time quantitative RT-PCR data: a model-based variance estimation approach to identify genes suited for normalization, applied to bladder and colon cancer datasets. Cancer Res 2004, 64:5245-5250.

6. Szabo A, Perou CM, Karaca M, Perreard L, Quackenbush JF, Bernard PS: Statistical modeling for selecting housekeeper genes. Genome Biol 2004, 5:R59.

7. Abruzzo LV, Lee KY, Fuller A, Silverman A, Keating MJ, Medeiros $L$, Coombes KR: Validation of oligonucleotide microarray data using microfluidic low-density arrays: a new statistical method to normalize real-time RT-PCR data. BioTechniques 2005, 38:785-792.

8. Rosic NN, Pernice M, Dunn S, Dove S, Hoegh-Guldberg O: Differential Regulation by Heat Stress of Novel Cytochrome P450 Genes from the Dinoflagellate Symbionts of Reef-Building Corals. Appl Environ Microbiol 2010, 76(9):2823-2829.

9. Li F, Luan W, Zhang C, Zhang J, Wang B, Xie Y, Li S, Xiang J: Cloning of cytoplasmic heat shock protein 90 (FCHSP90) from Fenneropenaeus chinensis and its expression response to heat shock and hypoxia. Cell Stress Chaperones 2009, 14(2):161-172.

10. Elekonich M: Extreme thermotolerance and behavioral induction of 70$\mathrm{kDa}$ heat shock proteins and their encoding genes in honey bees. Cell Stress Chaperones 2009, 14(2):219-226.

11. de Boer M, de Boer T, Marien J, Timmermans M, Nota B, van Straalen N, Ellers J, Roelofs D: Reference genes for QRT-PCR tested under various stress conditions in Folsomia candida and Orchesella cincta (Insecta, Collembola). BMC Mol Biol 2009, 10(1):54.. 
12. Xu M, Miller MS: Determination of murine fetal Cyp1a1 and 1b1 expression by real-time fluorescence reverse transcription-polymerase chain reaction. Toxicol Appl Pharmacol 2004, 201(3):295-302.

13. Lampo E, Van Poucke M, Vandesompele J, Erkens T, Van Zeveren A, Peelman L: Positive correlation between relative mRNA expression of PRNP and SPRN in cerebral and cerebellar cortex of sheep. Mol Cell Probes 2009, 23(1):60-64.

14. Tichopad A, Didier A, Pfaffl MW: Inhibition of real-time RT-PCR quantification due to tissuespecific contaminants. Mol Cell Probes 2004, 18:45-50.

15. Solanas M, Moral R, Escrich E: Unsuitability of Using Ribosomal RNA as Loading Control for Northern Blot Analyses Related to the Imbalance between Messenger and Ribosomal RNA Content in Rat Mammary Tumors. Anal Biochem 2001, 288(1):99-102.

16. Tricarico C, Pinzani P, Bianchi S, Paglierani M, Distante V, Pazzagli M, Bustin SA, Orlando C: Quantitative real-time reverse transcription polymerase chain reaction: normalization to rRNA or single housekeeping genes is inappropriate for human tissue biopsies. Anal Biochem 2002, 309(2):293-300.

17. Vincent S, Marty L, Fort P: S26 ribosomal protein RNA: an invariant control for gene regulation experiments in eucaryotic cells and tissues. Nucleic Acids Res 1993, 21(6):1498.

18. Zhang Z, Harrison P, Gerstein M: Identification and Analysis of Over 2000 Ribosomal Protein Pseudogenes in the Human Genome. Genome Res 2002, 12:1466-1482.

19. Zhang Z, Zheng D: Pseudogene evolution in the human genome. John Wiley \& Sons. Ltd: Chichester; 2008

20. Balogh A, Paragh G, Juhász A, Köbling T, Törocsik D, Mikó E, Varga V, Emri G, Horkay I, Scholtz B, et al: Reference genes for quantitative real time PCR in UVB irradiated keratinocytes. J Photochem Photobiol B 2008, 93(3):133-139.

21. Lo AS-Y, Liew C-T, Ngai S-M, Tsui SK-W, Fung K-P, Lee C-Y, Waye MM-Y: Developmental regulation and cellular distribution of human cytosolic malate dehydrogenase (MDH1). J Cell Biochem 2005, 94(4):763-773.

22. Setiawan A, Lokman PM: The use of reference gene selection programs to study the silvering transformation in a freshwater eel Anguilla australis: a cautionary tale. BMC Mol Biol 2010, 11(1):75.

23. Mehta R, Birerdinc A, Hossain N, Afendy A, Chandhoke V, Younossi Z, Baranova A: Validation of endogenous reference genes for qRT-PCR analysis of human visceral adipose samples. BMC Mol Biol 2010, 11(1):39.

24. de Kok JB, Roelofs RW, Giesendorf BA, Pennings JL, Waas ET, Feuth T, Swinkels DW, Span PN: Normalization of gene expression measurements in tumor tissues: comparison of 13 endogenous control genes. Lab Invest 2005, 85(1):154-159.

25. Vascotto SG, Beug S, Liversage RA, Tsilfidis C: Nvbeta-actin and NvGAPDH as normalization factors for gene expression analysis in limb regenerates and cultured blastema cells of the adult newt, Notophthalmus viridescens. Int J Dev Biol 2005, 49(7):833-842.

26. Bustin $\mathrm{S}$ : Absolute quantification of mRNA using real-time reverse transcription polymerase chain reaction assays. J Mol Endocrinol 2000, 25(2):169-193.

27. Rasmussen R: Quantification on the LyghtCycler. In Rapid Cycle Real-time PCR, Methods and Applications. Edited by: Meuer S, Wittwer C, Nakagawara K. Springer Press, Heidelberg; 2001:21-34.

28. Pfaffl MW, Horgan GW, Dempfle L: Relative expression software tool $\left(\right.$ RESTA $\left.\hat{A}^{\circ}\right)$ for group-wise comparison and statistical analysis of relative expression results in real-time PCR. Nucleic Acids Res 2002, 30(9):e36.

doi:10.1186/1471-2199-12-36

Cite this article as: Serrano et al: Use of Maximum Likelihood-Mixed Models to select stable reference genes: a case of heat stress response in sheep. BMC Molecular Biology 2011 12:36.

\section{Submit your next manuscript to BioMed Central and take full advantage of:}

- Convenient online submission

- Thorough peer review

- No space constraints or color figure charges

- Immediate publication on acceptance

- Inclusion in PubMed, CAS, Scopus and Google Scholar

- Research which is freely available for redistribution

Submit your manuscript at www.biomedcentral.com/submit
Ciomed Central 